\title{
Clinical Intestinal Transplantation
}

\author{
S. Todo, A. Tzakis, K. Abu-Elmagd, J. Reyes, H. Furukawa, B. Nour, J.J. Fung, and T.E. Starzl \\ Pittsburgh Transplant Institute, Department of Surgery, University of Pittsburgh, Pittsburgh, \\ Pennsylvania
}

We recently reported that clinical intestinal transplantation was feasible in 17 patients because of improved immunosuppression with FK 506 and refined monitoring of graft rejection. ${ }^{1}$ Described herein is the further follow-up of these 17 patients, and the clinical course of an additional 13 cases of intestinal transplantation performed thereafter.

\section{PATIENTS AND METHODS}

\section{Case Material}

During a two-and-a-half-year period, from May 1990 through November 1992. 30 patients underwent intestinal transplantation at our center. Fifteen patients were children and 15 were adults. Age, indication for transplantation, type of transplantation, and outcome are listed in Tables 1 and 2. Preoperatively, all of the patients were maintained by total parenteral nutrition (TPN) from 1 to 132 months and experienced various TPN-related complications such as sepsis, mineral deficiency, cholelithiasis, cholestasis, and portal hypertension.

All of the grafts were obtained from cadaveric donors of similar size and the same blood type as the recipient. Grafts were preserved with University of Wisconsin solution for a mean duration of 7.7 hours (ranging from 2.8 to 11.1 hours). No immunomodulation, either by administration of anti-lymphocyte globulin or irradiation, of the donor or graft was carried out.

Three different methods of intestinal transplantation were employed: isolated small bowel graft (SB), combined small bowel and liver graft (SB/L), or a composite of abdominal organs (multivisceral graft, MV). The type of transplantation procedure used depended on the cause of intestinal failure and the severity of extra-enteric organ failure, especially the liver. An isolated small bowel graft consisted of the entire intestine, except for short segments proximal to the ligament of Treitz and distal to the ileocecal valve. Nine patients ( 3 children and 6 adults) who had intestinal failure but normal liver function received an isolated small bowel graft. Seventeen patients ( 11 children and 6 adults) who had intestinal and liver failure received a combined small bowel and liver graft. The liver and small bowel were transplanted simultaneously and in continuity with the portal vein and their central arteries. Abdominal multivisceral transplantation was performed in 3 adult patients who had thrombotic abnormalities at the celiac axis (CA) and superior mesenteric artery (SMA), and in one child who had an extensive motility disorder throughout her entire gastrointestinal tract (from the stomach to the rectum). The multivisceral grafts included the stomach, liver, pancreas, and small bowel, and on one occasion the colon as well. Surgical procedures of graft procurement and transplantation were described before. ${ }^{2-4}$

Postoperative immunosuppressive therapy of intestinal transplant recipients was similar to the therapy for liver transplant recipients. Briefly, intravenous FK $506(0.1 \mathrm{mg} / \mathrm{kg}$ per day) was started immediately after the graft was revascularized, and was continued until oral dosing of 
FK $506(0.3 \mathrm{mg} / \mathrm{kg}$ per day) was feasible. Oral FK 506 was begun when patients were able to tolerate enteral feeding. A bolus injection of steroids was given intraoperatively and followed by a tapering of steroids thereafter. Prostaglandin E1 (0.6 to $0.8 \mu \mathrm{g} / \mathrm{kg}$ per hour) was given for 5 to 25 days postoperatively, and Imuran was given as a immunosuppressive supplement to patients for whom sufficient doses of FK 506 were hard to maintain.

Monitoring and treatment of intestinal graft rejection was done as previously described, in which careful evaluation of clinical course, endoscopic finding, and histopathologic study of endoscope-guide biopsies were essential. ${ }^{1}$ Enteral feeding was usually started from 1 to 2 weeks (average of 17 days) posttransplant and augmented gradually as TPN was decreased. To prevent bacterial translocation, selective decontamination of the gastrointestinal tract was continued for 4 to 8 weeks posttransplant.

\section{RESULTS}

\section{Isolated Small Bowel Transplantation}

Of the 9 SB recipients, $8(88.8 \%)$ are alive from 8 to 15 months. One patient died of overwhelming sepsis 71 days after retransplantation. This patient was retransplanted after 22 months for drug-noncompliant rejection. Another graft had to be removed at 8 months because of acute rejection: immunosuppression was stopped due to demyelination of the white matter of the brain. The patient was restarted on TPN. Currently, 7 of the 10 grafts ( 9 primary grafts and 1 retransplanted graft) are functioning. All 7 of the patients with functioning grafts are maintaining their nutritional requirements without TPN support.

\section{Combined Liver and Small Bowel Transplantation}

Thirteen of the $17 \mathrm{SB} / \mathrm{L}$ recipients (76\%) are alive from 5 to 30 months posttransplant. Four pediatric patients died: 3 due to sepsis and 1 due to postoperative lymphoproliferative disease. Technical complications (intestinal anastomotic leakage, biliary anastomotic leakage, or hepatic artery thrombosis) were the cause of the lethal septic complications in the 3 pediatric patients. In addition to the technical complication, 1 patient that died 23 days posttransplant was also found to have graft-versus-host disease (GVHD). Immunohistochemical examination of skin biopsies revealed GVHD at 22 days, but not at 19 days. All of the 13 surviving recipients, except for one, are completely free from TPN.

\section{Abdominal Multivisceral Transplantation}

Abdominal multivisceral transplantation was performed in 4 patients (1 pediatric patient and 3 adults). All 4 of the patients are alive with functioning grafts from 3 to 15 months.

\section{COMMENTS}

Intestinal transplantation is a theoretical choice of treatment for patients who have irreversible intestinal failure. However, except for a few isolated cases of success, rejection, technical problems, sepsis, and poor graft preservation have prevented successful results in the more than two dozen attempts at small bowel transplantation under conventional immunosuppression. ${ }^{5-7}$ The major obstacle in the past was the inability to control and to monitor graft rejection. The intestine is thought to induce graft rejection more vigorously than other organs, because it has abundant lymphoreticular cells in the Peyer's patches, lamina propria, and mesenteric lymph nodes. Although rejection of the liver and kidney can be estimated by the elevation of bilirubin and creatinine, respectively, there is no biochemical marker of intestinal damage. In our experience with 30 small bowel transplant recipients, a new and potent immunosuppressant, FK 506, was used for posttransplant prevention: control of rejection and histopathologic analysis of endoscope-guided biopsies was used for monitoring 
of graft rejection. As shown with the transplantation of the liver, kidney, heart, or lung, ${ }^{8} \mathrm{FK}$ 506 is also able to exhibit its potent anti-rejection effect on the intestine. Contrary to prior assumptions on the unreliability of pathologic diagnosis of small bowel rejection, endoscopeguided mucosal biopsies of the small bowel graft and clinical impression offered sufficient information to diagnose rejection.

Although the indications need to be carefully appraised according to individual cases, three different types of intestinal transplantation can be performed. Isolated small bowel transplantation is indicated for patients who have problems only with their intestine. Combined liver and small bowel transplantation is indicated for patients who have both liver and intestinal failure. Multivisceral transplantation is indicated for patients who have extensive thrombotic disorders involving both the celiac axis and superior mesenteric artery or possibly the splanchnic system. Multivisceral transplantation may also be indicated for patients who have a severe motility disorder that involves the entire gastrointestinal tract.

Although a much longer follow-up and a refinement of postoperative management are required, our experience with these 30 recipients suggests that intestinal transplantation is becoming a practical reality.

\section{Acknowledgments}

This work was supported by research grants from the Veterans Administration and by Project Grant No. OK 29961 from the National Institutes of Health, Bethesda, Maryland.

\section{References}

1. Todo S, Tzakis AG, Abu-Elmagd K, et al. Ann Surg 1992;216:223. [PubMed: 1384443]

2. Starzl TE, Todo S, Tzakis A, et al. Surg Gynecol Obstet 1991;172:335. [PubMed: 2028370]

3. Casavilla A, Selby R, Abu-Elmagd K, et al. Ann Surg 1992;216:85.

4. Tzakis AG, Todo S, Reyes J, et al. Surg Gynecol Obstet 1993;176:297. [PubMed: 8438205]

5. Grant D, Wall W, Mimeault R, et al. Lancet 1990;335:181. [PubMed: 1967664]

6. Deltz E, Schroeder P, Gebhardt H, et al. Clin Transpl 1989;3:89.

7. Goulet O, Revillon Y, Brousse N, et al. Transplantation 1992;53:940. [PubMed: 1533072]

8. Starzl TE, Fung JJ, Todo S, et al. Transplant Proc 1991;23(6) 
